\title{
Association Study of Sperm DNA Integrity, Routine Semen Parameters and In Vitro Fertilization Clinical Outcome
}

\section{Yunzhu Lan ( $\nabla$ lanyunzhu1990@163.com )}

The Affiliated Hospital of Southwest Medical University https://orcid.org/0000-0001-7137-0410

\section{Xinjian Feng}

Luzhou's people hospital

\section{Xingyu Sun}

The Affiliated Hospital of Southwest Medical University

\section{Li Fu}

The Affiliated Hospital of Southwest Medical University

\section{Research}

Keywords: sperm DNA integrity, SCD, semen routine, in vitro fertilization

Posted Date: May 26th, 2021

DOI: https://doi.org/10.21203/rs.3.rs-549300/v1

License: (c) (i) This work is licensed under a Creative Commons Attribution 4.0 International License. Read Full License 


\section{Abstract}

This paper explores the relationship among sperm DNA integrity, routine semen parameters and in vitro fertilization (IVF) clinical outcome. It applies sperm chromatin dispersion (SCD) test to conduct sperm DNA fragmentation index (DFI) towards the semen samples of 60 male patients who undergoing assisted reproduction techniques (ART) treatments including intrauterine insemination (IUI), in vitro fertilization (IVF), and intracytoplasmic sperm injection (ICSI) treatment in human assisted reproductive medical technology of our hospital. According to the test results, semen samples are divided into group $A$ (DFI $\leq 10 \%)$, group $B(10 \%<D F \mid<30 \%)$ and group $C(D F I \geq 30 \%)$ to compare semen routine main parameters in each group as well as fertility rate, cleavage rate and high-quality embryonic rate. There is a negative correlation among DFI and sperm activate rate, progressive motility(PR) rate as well as non-progressive motility(NP) rate. The difference of the sperm activate rate, progressive motility rate and non-progressive motility rate in three groups are statistically significant $(P<0.05)$, and there is no statistically significant difference in sperm concentration ( $P>0.05$ ). The fertility rate, cleavage rate and high-quality embryonic rate in each group have no statistical significance ( $P>0.05)$. Sperm DNA integrity have some connection with routine semen parameters, thus, it could play a guiding role for the analysis of semen routine diagnosis. DFI has little influence on the assisted reproductive technology IVF clinical outcome, which requires follow-up tracking clinical outcome and a large number of samples for validation.

\section{Introduction}

Approximately $60-80$ million people suffer infertility around the world, which is almost $10 \%-15 \%$ of the bearing population ${ }^{[1]}$. Among these infertile couples, about $20 \%$ of cases are associated with male infertility ${ }^{[2]}$. However, the semen routine analysis shows that about $15 \%$ infertile men still have normal semen ${ }^{[3]}$. As the sperm DNA carries with parents' genetic information, the examination of sperm DNA integrity not only can make us have a more comprehensive understanding of the sperm function in male patients, but also provide reference frame for assisted reproductive technology outcome prediction. Under

normal circumstances, ovums are able to identify and repair damaged sperms by repairing function $[4,5]$. As a woman gets older, there come adverse conditions including ovary dysfunction, organic diathesis decline and the ovum self-repair function gradually reduces. When overburden, it may exert some effect on the outcome of assisted reproductive technology. Therefore, sperm DNA integrity is worth studying to assess male fertility and explore the relationship between assisted reproductive technology clinical outcomes.

At present, there are mainly two methods for the determination of DFI : sperm chromatin structure assay ( SCSA ) and sperm chromatin dispersion ( SCD ).Studies have shown that SCSA is determined by the machine sperm DFI, with objectivity, however, due to the need to buy high flow cytometry, its cost is too high, ordinary medical institutions are difficult to promote. Secondly, there are limitations for flow cytometry to detect samples with extremely low concentration. If the concentration is particularly low and the detection time is long, the number of detected sperm cannot meet the detection requirements of flow 
cytometry. Compared with SCSA, SCD depends on human judgment ${ }^{[6]}$. Although SCD has subjective influence, it is simple to operate and can maintain the same detection level for low concentration semen samples. Therefore, this experiment mainly used SCD method to detect DFI, and explored the correlation between DFI and routine semen parameters and clinical outcomes of in vitro fertilization.

\section{Material And Methods}

\subsection{Patients}

The research object of this study was 60 infertile couples who undergoing assisted reproduction techniques (ART) treatments including intrauterine insemination (IUI), in vitro fertilization (IVF), and intracytoplasmic sperm injection (ICSI) treatment in human assisted reproductive medical technology of southwest medical university from May 2019 to May 2020. Inclusion criteria: after regular sexual life for more than one year, there was no natural conceived on the condition of no contraception. The female showed normal ovarian function. Neither of the couple show any inherited disorders, sexual dysfunction and anatomical deformity, mumps history. There were no obvious abnormalities can be found in physical examination and ancillary testing. Male patients preservde pre-treatment sperm samples for routine semen analysis and sperm nuclear DNA integrity detection. For female participants, they were arranged ovums extraction under the ultrasound detection to conduct IVF assisted reproductive technology treatment. Based on DFI, the participants were divided into group $A$ (DFI $\leq 10 \%)$, group $B(10 \%<D F I<30 \%)$ and group $\mathrm{C}(\mathrm{DFI} \geq 30 \%)$.

\subsection{Semen routine}

After keeping abstinence for 2-7 days, samples were left in the one-time sperm collectors through masturbation and stored in the environment of $20-37^{\circ} \mathrm{C}$ with the specimen number, name of patients, semen collection time and days without ejaculation record. It kept notes of total specimens and liquefied duration. According to the Human Semen Examination and Processing Laboratory Manual (version V) published by Word Health Organization in 2010 [7] , it applied computer-assisted sperm, microbial dynamic (static) state image analysis system (THTF, CASAS-QH-III) to analysis and record the following data: sperm concentration, sperm activate rate, sperm non- progressive motility rate, progressive motility. rate and immotility rate.

\subsection{SCD}

This study improved the SCD method proposed by scholars such as Meseguer ${ }^{[8]}$. In this study, it first used phosphate buffer (PBS pH 7.2) to wash semen samples adequately. After centrifugation the supernatant was discarded. The concentration of the specimen was adjusted to $4-10 \times 106 / \mathrm{ml}$. Make $1 \% 100 \mathrm{ml} \mathrm{low}$ melting-point agarose solution, took 70 ul to $1.5 \mathrm{ml}$ centrifuge tube to be melted in $65-90^{\circ} \mathrm{C}$ water bath and be kept in $47^{\circ} \mathrm{C}$ for three minutes. Added treated semen samples $30 \mathrm{ul}$ and do mechanical blending. Dropped $20 \mathrm{ul}$ into the slide with $0.65 \%$ prepared standard agarose gel, closed the $18 \times 18 \mathrm{~cm}$ cover glass to be placed in $4^{\circ} \mathrm{C}$ refrigerator for 35 minutes. If the agarose was completely cured, carefully peeled off 
the surface cover glass. Put into the prepared diluted hydrochloric acid $(0.08 \mathrm{mmol} / \mathrm{L})$ with avoiding light in order to degeneration and immerse in the sperm cracking liquid $(0.4 \mathrm{~mol} / \mathrm{L}$ DTT consists of Tris, DTT, SDS and EDTA). Conducted natural dehydration in $70 \%, 90 \%, 100 \%$ ethanol and withdraw them into object slide stand to dry naturally for Wright Stain. After completion of dyed, the samples were placed under Olympus microscope $(10 \times 40)$ to observe the account. It was worth stressing that more emphasis was placed on the preparation of reagent when using on the basis of previous test, especially sperm cracking liquid configuration.

Because normal sperm DNA could be attached to the loose chromatin structure and form a dizzy ring, while damaged sperm DNA cannot or can only form a small ring ${ }^{[9]}$. Through microscope observation, sperm head diameter is $\mathrm{d} 1$ and unilateral halo thickness is $\mathrm{d} 2$ (see Fig 1 ). When evaluating $\mathrm{d} 2 \leq 1 / 3 \mathrm{~d} 1$, it showed that the existence of sperm DNA fragmentation (small halo ring); if the head was deeply stained, it was considered as no halo ring. As can be seen from Fig 2 , big halo ring ( $d 2 \geq 2 / 3 d 1)$ and middle halo ring $(1 / 3 \mathrm{~d} 1<\mathrm{d} 2<2 / 3 \mathrm{~d} 1)$ were complete DNA sperm.

\subsection{Conventional stimulation protocol Fertilization}

On day 2 of menstrual cycle, the female participants began to accept intramuscular injectiongonadotrophin releasing hormone agonists (Diphereline, Ipsen Pharma Biotech) $0.1 \mathrm{mg} /$ day for 14 consecutive days. B ultrasound and estrogen were used to monitor the condition of follicular development at any time. After reaching the standard of falling tone, gave with recombinant human follicle stimulating hormone injections (Recombinant Human Follitropin for Injection, Swiss Serono pharmaceutical). When the $18 \mathrm{~nm}$ above follicles were more than 2 , combined with patient blood $\mathrm{P}$ and E2 level, they were given intramuscularly injection of human chorionic gonadotropin (HCG, Swiss Serono Company) 6000U. 36 hours after intramuscularly injection, punctured ovum extraction under the guide of B ultrasound. Added Protein insemination culture (G-IVF PLUS, vitrolife, Sweden) and placed in $37^{\circ} \mathrm{C}, 6 \%$ $\mathrm{CO}_{2}$ incubator to cultivate sperm egg interaction for five hours.

\subsection{Embryological observation and high-quality embryo number selection}

Normal fertilization refered to 2 pronuclears (PN) and Polar body2 (Pb2) could be found under a microscope. Abnormal fertilization refered to monopronuclear (1PN) was less than or equal to 1 or multipronuclear (3PN) was equal or greater than 3. According to Peter's cleavage stage ${ }^{[10]}$ embryo scoring system, embryo quality could be assessed through segmentation sphere volume, form, fragments and cytoplasma granula.

Those I and II level embryos that develoed into 6-10 segmentation spheres on the third day were defined as high quality embryos, and the rest were considered as low-score embryos. High quality embryos were selected to be transplanted.

\subsection{Data collection}


The study collected the data of clinical sperm concentration, sperm activate rate, sperm progressive motility rate, non-progressive motility rate and immotility rate (see Figure 3). SCD method was used to calculate the sperm number with DNA fragmentation in 400 sperm smears under microscope. Sperm DNA fragmentation index $(D F I)=($ small halo ring sperm+ no halo ring sperm $) /$ observed 400 sperm $\times 100 \%$

[11]. Figure 4 shows retrieved oocytes, fertilized oocytes, cleavage number and high quality embryo number after the treatment of IVF assisted reproductive technology. Fertility rate=fertilized ocum number/mature ocum number $\times 100 \%$, cleavage rate=cleavage number/fertilized ocum number $\times 100 \%$, high-quality embryo rate=high quality embryo number/total number of embryos $\times 100 \%$.

\subsection{Statistical analysis}

SPSS17.1 statistical software was applied to deal with all the collected statistics analysis. All the data adopted mean standard deviation and measurement data using to test analysis. One-way analysis of variance was used on the basis of DFI classification. It explored the relationship between DFI and routine semen parameters, fertility rate, cleavage rate and high-quality embryo rate. Correlation analysis used Pearson correlation analysis and the correlation coefficient refers to $P<0.05$ presents the difference was statistically significant.

\section{Results}

3.1 Statistical analysis of each group and routine semen parameters

After welch test correction, there were significant differences between sperm activate rate, progressive motility rate, non-progressive motility rate and immotility rate under DFI classification $(P<0.05)$. It had statistical significance. However, sperm concentration had no significant difference $(P>0.05)$. It was shown that the differences among sperm activate rate, progressive motility rate, non-progressive motility rate was greatest in group $A(D F I \leq 10 \%)$, followed with group $B(10 \%<D F \mid<30 \%)$ and group $C(D F I \geq 30 \%)$. The differences in spermatium rate showed group $\mathrm{C}>$ group $\mathrm{B}>$ group $\mathrm{A}$.(Table 1) Meanwhile, DFI and sperm activate rate, progressive motility rate, non-progressive motility rate had a negative correlation, which meant if the DFI was higher, sperm activate rate, progressive motility rate, non- progressive motility rate became lower; On the other hand, it showed a positive correlation with immotility rate. As can be seen from Table 2, if the DFI was higher, the proportion of immotility was higher correspondingly.

\subsection{Statistical analysis of DFI each group and IVF clinical outcome}

Through welch test correction, it could be seen from Table 2 that there was no statistical significance when comparing fertility rate, cleavage rate and high-quality embryo rate with DFI in each group after IVF assisted reproductive technology ( $P>0.05)$.

3.3 Correlation between each group and IVF clinical outcome 
Pearson correlation analysis was used to explore DFI and fertility rate, cleavage rate and high-quality embryo rate with represents correlation coefficient. The results showed that no correlation was observed among sperm fragmentation rate DFI and fertility rate, cleavage rate and high-quality embryo rate (See Table 4).

\section{Discuss}

It is still a matter of public debate whether sperm DFI can represent routine semen parameters to conduct clinical diagnosis towards partients'sperm quality. According to the semen routine parameters and DFI Pearson correlation analysis, it was found that DFI and sperm activate rate, progressive motility rate, nonprogressive motility rate has negative correlation, that is, if the DFI was higher, sperm activate rate, progressive motility rate, non- progressive motility rate were lower; Meanwhile, it has positive correlation with immotility. rate, which means higher DFI will have higher immotility. rate. The findings are in consistent with most of the DFI and routine semen parameters analysis researches. When analyzing the relationship between sperm DNA integrity and seminal parameters, Multiple previous analyses ${ }^{[12.13]}$ that sperm nucleus DFI shows negative correlation with forward movement sperm rate and fast movement sperm concentration. The findings ${ }^{[14]}$ show that sperm activate rate and $(a+b)$ level sperm rate of male after 40 reduce significantly with age.

The study divided DFI into group A (DFI $\leq 10 \%)$, group B $(10 \%<\mathrm{DFI}<30 \%)$ and group $\mathrm{C}(\mathrm{DFI} \geq 30 \%)$, and conducted One-Way ANOVA towards correspondent routine semen parameters. Considered the number of each group are different, it did homogeneity test of variances before one-way analysis of variance. After welch test correction, there were significant differences among sperm activate rate, non- progressive motility rate, progressive motility rate, and immotility. rate under DFI classification. But sperm concentration had no significant difference. It was shown that the differences among sperm activate rate, non- progressive motility rate, progressive motility rate, was greatest in group $A$ (DFI $\leq 10 \%)$, followed with group $B(10 \%<D F \mid<30 \%)$ and smallest in group $C(D F I \geq 30 \%)$. Moreover, the differences in spermatium rate showed group $\mathrm{C}>$ group $\mathrm{B}>$ group $\mathrm{A}$, which was in accordance with other study ${ }^{[15]}$. If the sperm is influenced by themselves or external environment high risk factors such as impairing of immunity, sleep deprivation, age and smoking, the highly condensed chromatin results inside the sperm will be destroyed [16] resulting in sperm chromatin osteoporosis, which finally makes the internal pattern of nucleoprotein abnormal and sperm DNA damage and fracture. At the same time, the production of reactive oxygen species (ROS) in the spermatid increases. Accumulation too much will lead to stronger oxygen free radical attack ${ }^{[17,18]}$. Early-stage sperm apoptosis usually uses Fas/FasL and caspase to mediate, however, when the way is abnormal, it can make the sperm DNA damage.

Part cell apoptosis will prompt sperm cellss to escape natural death, which further aggravates the sperm DNA damage [19]. Too much sperm DNA chain rupture will induce sperm cells apoptosis, which can be demonstrated in sperm morphology, sperm activate rate, sperm density and forward movement conditions. It is reported that the damage of sperm DNA integrity will also change sperm movement and 
sperm morphology ${ }^{[20,21]}$.Sperm motion device abnormal can also result in the decrease of sperm vitality, meanwhile, it will have direct effect on sperm nuclear DNA, causing spontaneous damage of sperm DNA, sperm DNA fragments rate to increase ${ }^{[22]}$. After the sperm goes through the cervical mucus, it touches eggs zona pellucida for fusion and complete the fertilization step. In the process, sperm forward movement rate and normal morphology are the assurance of fertilization to be completed.

The results of this study shows that fertility rate, cleavage rate and high-quality embryo rate have no significant differences under DFI classification ( $P>0.05$ ). Pearson correlation analysis indicates sperm fragmentation rate has no association with fertility rate, cleavage rate and high-quality embryo rate. It is believed that high DFI and low DFI group show no significant relationship with fertility rate. In other words, whether DFI is high or low has no influence on fertility rate ${ }^{[23]}$. Whether DFI will affect the analysis of assisted reproductive technologyclinical outcome, domestic and foreign scholars have different research conclusions. The author thinks that, in principle, sperm DFI has little influence on the clinical outcome of IVF-ET and ICSI, especially little effect on IVF-ET. ICSI seminal fluid technology is directly into the egg cell, there is no egg zona pellucida recognition process. In addition, eggs can repair DNA damaged sperm cells. Only when the repaire is overloaded, it will exert some influence on the clinical outcome of ICSI. But before the success of IVF, it requires egg zona pellucida for screening application, to a certain extent, the sperm that successfully fertilized has relatively small damage, so sperm DFI has little or no influence on the clinical outcome of IVF ${ }^{[24,25]}$. Besides that, egg maternal gene may dominate an important position in the process of embryonic development for regulating the fertilization process and embryonic development process, which makes egg DNA integrity become more important in the early embryonic development ${ }^{[26]}$. In the study of male patients whose 6 gene promoter methylation will make sperm protamine abnormal, sperm DFI and IVF outcome has no association. When exploring the relationship between IVF and perinatal morbidity rate, some studies have previously posited that the level of DFI has no obvious effect on the clinical outcome of IVF ${ }^{[27]}$. After analyzing sperm DNA fragmentation rate, the regardless of own eggs or donor eggs, the level of DFI does not achieve statistical significance with fertility rate, available embryos rate and clinical pregnancy rate, which is in consistent with the conclusion of this study, that is, there are no significant differences in fertility rate, cleavage rate, highquality embryo rate under DFI classification ${ }^{[28]}$.

DFI can be used as a supplement of semen routine analysis' clinical diagnosis. DFI has no effect or little effect on IVF clinical outcome, but cannot rule out whether it is connected with the following clinical outcome. Thus, it can continue to follow-up, record clinical outcome data including the pregnancy rate, miscarriage rate and aberration rate for DFI statistical analysis.

\section{Declarations}

\section{Authors' contributions}

Yun-zhu Lan: Contributed to study concept and design, acquisition of data, analysis and interpretation of data, and drafting of the manuscript, and has full access to the data in the study and takes responsibility 
for the integrity of the data and the accuracy of the data analyses.

Xin-jian Feng: Contributed to study concept and design, analysis and interpretation of data, study supervision.

Xing-yu Sun: Contributed to data analysis and editing of the manuscript, and provided expertise.

Li Fu: reviewed and edited the manuscript

\section{Competing interests}

The authors declare that they have no conflict of interest.

\section{Funding}

The study was supported by Science Foundation of Southwest Medical University (no. 2016217)and the affiliated hospital of southwest medical university(2014-71-17).

\section{Ethics approval and consent to participate}

The studies involving human participants were reviewed and approved by Ethics Committee of Affiliated Hospital of Southwest Medical University. Written informed consent for participation was not required for this study in accordance with the national legislation and the institutional requirements.

\section{Consent for publication}

Written informed consent for publication was obtained from all participants.

\section{Availability of data and material}

There are no linked research data sets for this paper. Data will be made available on request.

\section{Provenance and peer review}

This article was not commissioned and was externally peer reviewed.

\section{Acknowledgments}

We thank the participants of the survey and all staff members involved in this study for their painstaking efforts in conducting the data collection. We are grateful to the Affiliated Hospital of Southwest Medical University for funding and to the women who provided the survey data.

\section{References}

1. Saradha Baskaran, Chak-Lam Cho,Role of Sperm DNA Damage in Male Infertility Assessment.Male Infertility in Reproductive Medicine.2019:57-68.https://doi.org/10.1201/9780429485763-7 
2. Sperm DNA Damage, ART Outcomes, and Laboratory Methods for Selecting DNA Intact Sperm for ICSI,Male Infertility,2020:717-734https://doi.org/10.1007/978-3-030-32300-4_58

3. Cao XW1, Lin K, Li CY, Yuan CW..A review of WHO Laboratory Manual for the Examination and Processing of Human Semen (5th edition)[J].Zhonghua Nan Ke Xue. 2011 Dec;17(12):1059-63.

4. Ahmad Majzoub, Ashok Agarwal etal.Clinical utility of sperm DNA damage in male infertility.Panminerva Medica.2019.61(2).https://doi.org/10.23736/s0031-0808.18.03530-9

5. Russell S. Kirby.Assisted Reproductive Technology and Developmental Outcomes.Pediatrics.2018.142(6): e20183072.https://doi.org/10.1542/peds.2018-3072

6. Hongbo Cheng, Mutian Han,et al.Importance of a semen analysis report for determining the relationship between SCSA sperm DNA fragmentation index and assisted reproductive technology pregnancy rate.Reproductive Biology.2020.20(4):460-464.

https://doi.org/10.1016/j.repbio.2019.09.002

7. W. C. L. Ford.World Health Organization.Comments on the release of the 5th edition of the WHO Laboratory Manual for the Examination and Processing of Human Semen[J].Asian Journal of Andrology.2010.12(1):59-63.https://doi.org/10.1038/aja.2008.57

8. M. Meseguer, R. Santiso, N. The effect of cancer on sperm DNA fragmentation measured by the sperm chromatin dispersion (SCD) test.Fertility and Sterility.2007.88:S337.https://doi.org/10.1016 /j.fertnstert.2007.07.1125

9. Tianyi Feng, Jamie Gosálvez,et al.Sperm chromatin dispersion test (SCDt) for the assessment of sperm DNA fragmentation in black tiger prawn, Penaeus monodon.Aquaculture.2018.491:281-288. https://doi.org/10.1016/j.aquaculture.2018.03.041

10. Peter Brinsden. A textbook of in vitro fertilization and assisted reproduction[M]. New York: The Parthenon Publishing Group Inc, 2005.https://doi.org/10.1201/9780367804305

11. Craig Niederberger.Relationship between Sperm Aneuploidy, Sperm DNA Integrity, Chromatin Packaging, Traditional Semen Parameters, and Recurrent Pregnancy Loss. Journal of Urology.2016.196(4):1232.https://doi.org/10.1016/j.juro.2016.07.017

12. Simon L, Lewis SE. Sperm DNA damage or progressive motility: which one is the better predictor of fertilization in vitro? Syst Biol Reprod Med 2011;57:133-8. https://doi.org/10.3109/19396368.2011.553984

13. Peter Zarén, Sara Alson.Interaction between serum levels of Anti-Mullerian Hormone and the degree of sperm DNA fragmentation measured by sperm chromatin structure assay can be a predictor for the outcome of standard in vitro fertilization.PLOS ONE.2019.14(8):e0220909.https://doi.org/10.1371/journal.pone.0220909

14. Xu Tingyun, Hu Jiabo, Gao Huasheng et al. Semen quality analysis of 2640 male infertility patients in Suzhou [J]. National Journal of Andrology, 2011, 17(6): 511-515.

15. Jan Tesarik.MSOME and Sperm DNA Integrity: Biological and Clinical Considerations.Non-Invasive Sperm Selection for In Vitro Fertilization.2015:137 to 147.https://doi.org/10.1007/978-1-4939-1411$1 \_14$ 
16. Preben Christensen, Anders Birck.Assessment of Sperm DNA Integrity and Implications for the Outcome of ICSI Treatments.Intracytoplasmic Sperm Injection.2018:6384.https://doi.org/10.1007/978-3-319-70497-5_6

17. Robert John Aitken.Nitroblue tetrazolium (NBT) assay.Reproductive BioMedicine Online volume.2018.36(1):90-91.https://doi.org/10.1016/j.rbmo.2017.09.005

18. A. Ayaz, A. Agarwal,et al.Proteomic analysis of sperm proteins in infertile men with high levels of reactive oxygen species. National Institutes of Health American Center for Reproductive Medicine.https://doi.org/10.1111/and.13015

19. Sevilay Erimşah.Assessment of Sperm DNA İntegrity From Normozoospermic Infertile Men Selected by Different Selection Methods.Proceedings of the 15th International Congress of Histochemistry and Cytochemistry.2017.https://doi.org/10.5505/2017ichc.pp-162

20. Kristian Leisegang, Ralf Henkel.Oxidative Stress: Relevance, Evaluation, and Management.Male Infertility in Reproductive Medicine.2019:119-128.https://doi.org/10.1201/9780429485763-13

21. ERIK HøST, SVEND LINDENBERG, et al.DNA strand breaks in human spermatozoa: Correlation with fertilization in vitro in oligozoospermic men and in men with unexplained infertility. Acta Obstetricia et Gynecologica Scandinavica.2000.79(3):189-https://doi.org/10.1034/j.1600-

0412.2000.079003189.x

22. Erenpreiss J, Spano M, Erenpreisa J, et al.Sperm chromatin Structure and male fertility:biological and clinical aspects [J]. Asian J Androl, 2006, 8(1) : 11-29.https://doi.org/10.1111/j.1745-

7262.2006.00112.x

23. Violeta S. Rilcheva,et al.Meta-analysis of the relationship between sperm DNA integrity and assisted reproductive outcome.Journal of Biomedical and Clinical Research.2016.9(1):2129.https://doi.org/10.1515/jbcr-2016-0003

24. Wei Wu, Chuncheng Lu,et al.Lack of association between DAZ gene methylation patterns and spermatogenic failure.Clinical Chemistry and Laboratory Medicine.2010.48(3).https://doi.org/10.1515/cclm.2010.007

25. Russell S. Kirby.Assisted Reproductive Technology and Developmental Outcomes [J]. Pediatrics.2018.142(6)e20183072.https://doi.org/10.1542/peds.2018-3072

26. Marcos Meseguer, Rebeca Santiso,et al.Effect of sperm DNA fragmentation on pregnancy outcome depends on oocytmm $\mathrm{m} \quad$,...v...v..v.v..e quality.Fertility and Sterility.2011.95(1):124128.https://doi.org/10.1016/j.fertnstert.2010.05.055

27. Laszlo Nanassy, Douglas T Carrell.Analysis of the methylation pattern of six gene promoters in sperm of men with abnormal protamination.Asian Journal of Andrology.2011.13(12):342346.https://doi.org/10.1038/aja.2010.160

28. Marga Esbert, Alberto Pacheco, et al.Impact of sperm DNA fragmentation on the outcome of IVF with own or donated oocytes.Reproductive BioMedicine Online.23(5):704-

710.https://doi.org/10.1016/j.rbmo.2011.07.010 
29. Sevastiani Antonouli, Achilleas Papatheodorou,et al.The impact of sperm DNA fragmentation on ICSI outcome in cases of donated oocytes.Archives of Gynecology and Obstetrics.2019.300(1):207215.https://doi.org/10.1007/s00404-019-05133-9

\section{Tables}

Table 1 Different pieces of sperm rate DFI grouping IVF clinical outcome of single factor analysis of variance

\begin{tabular}{|c|c|c|c|c|c|c|c|}
\hline & & $\mathrm{N}$ & Average & Standard & $F$ & $P$ & T test \\
\hline & & & & Deviation & & & $P$ value \\
\hline \multirow[t]{4}{*}{ Fertilization rate } & $\mathrm{A}$ & 21 & 90.68 & 14.66 & 0.787 & 0.46 & 0.011 \\
\hline & B & 28 & 86.39 & 17.62 & & & \\
\hline & $\mathrm{C}$ & 11 & 82.00 & 28.09 & & & \\
\hline & Total & 60 & 87.08 & 18.93 & & & \\
\hline \multirow[t]{4}{*}{ Cleavage rate } & A & 21 & 93.22 & 22.61 & 1.22 & 0.303 & 0.011 \\
\hline & B & 28 & 98.54 & 4.86 & & & \\
\hline & $\mathrm{C}$ & 11 & 100.00 & 0.00 & & & \\
\hline & Total & 60 & 96.94 & 13.86 & & & \\
\hline High quality & A & 21 & 52.47 & 28.69 & 0.039 & 0.962 & 0.599 \\
\hline \multirow[t]{3}{*}{ embryo rate } & B & 28 & 50.34 & 34.93 & & & \\
\hline & $\mathrm{C}$ & 11 & 49.48 & 33.05 & & & \\
\hline & Total & 60 & 50.92 & 32.00 & & & \\
\hline
\end{tabular}


**. At 0.01 level (double side). *. At 0.05 level (double side).

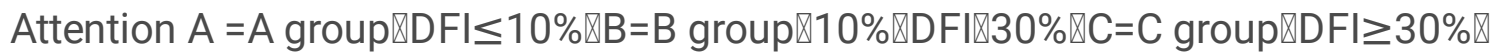

Table 2 Correlation analysis of DFI) between groups with IVF clinical outcome

\begin{tabular}{lcc}
\hline & correlation coefficent & $P$ Values \\
\hline Fertilization rate & -0.152 & 0.247 \\
Cleavage rate & 0.202 & 0.122 \\
High quality embryo rate & 0.004 & 0.974 \\
\hline
\end{tabular}

**. At 0.01 level (double side). *. At 0.05 level (double side).

Table 3 Different pieces of sperm rate DFI grouping IVF clinical outcome of single factor analysis of variance 


\begin{tabular}{|c|c|c|c|c|c|c|c|}
\hline & & $\mathrm{N}$ & Average & Standard & $F$ & $P$ & $\mathrm{~T}$ test \\
\hline & & & & Deviation & & & $P$ value \\
\hline \multirow[t]{4}{*}{ Fertilization rate } & $\mathrm{A}$ & 21 & 90.68 & 14.66 & 0.787 & 0.46 & 0.011 \\
\hline & B & 28 & 86.39 & 17.62 & & & \\
\hline & $\mathrm{C}$ & 11 & 82.00 & 28.09 & & & \\
\hline & Total & 60 & 87.08 & 18.93 & & & \\
\hline \multirow[t]{4}{*}{ Cleavage rate } & $\mathrm{A}$ & 21 & 93.22 & 22.61 & 1.22 & 0.303 & 0.011 \\
\hline & B & 28 & 98.54 & 4.86 & & & \\
\hline & $\mathrm{C}$ & 11 & 100.00 & 0.00 & & & \\
\hline & Total & 60 & 96.94 & 13.86 & & & \\
\hline High quality & $\mathrm{A}$ & 21 & 52.47 & 28.69 & 0.039 & 0.962 & 0.599 \\
\hline \multirow[t]{3}{*}{ embryo rate } & B & 28 & 50.34 & 34.93 & & & \\
\hline & $\mathrm{C}$ & 11 & 49.48 & 33.05 & & & \\
\hline & Total & 60 & 50.92 & 32.00 & & & \\
\hline
\end{tabular}

**. At 0.01 level (double side). *. At 0.05 level (double side).

Attention $A=A$ group $₫ D F I \leq 10 \% \rrbracket B=B$ group $₫ 10 \% \otimes D F I \otimes 30 \% \otimes C=C$ group $(D F I \geq 30 \%)$

Table 4 Correlation analysis of DFI between groups with IVF clinical outcome 


\begin{tabular}{lcc}
\hline & correlation coefficent & $P$ Values \\
\hline Fertilization rate & -0.152 & 0.247 \\
Cleavage rate & 0.202 & 0.122 \\
High quality embryo rate & 0.004 & 0.974 \\
\hline
\end{tabular}

* *. At 0.01 level (double side). *. At 0.05 level (double side).

\section{Figures}

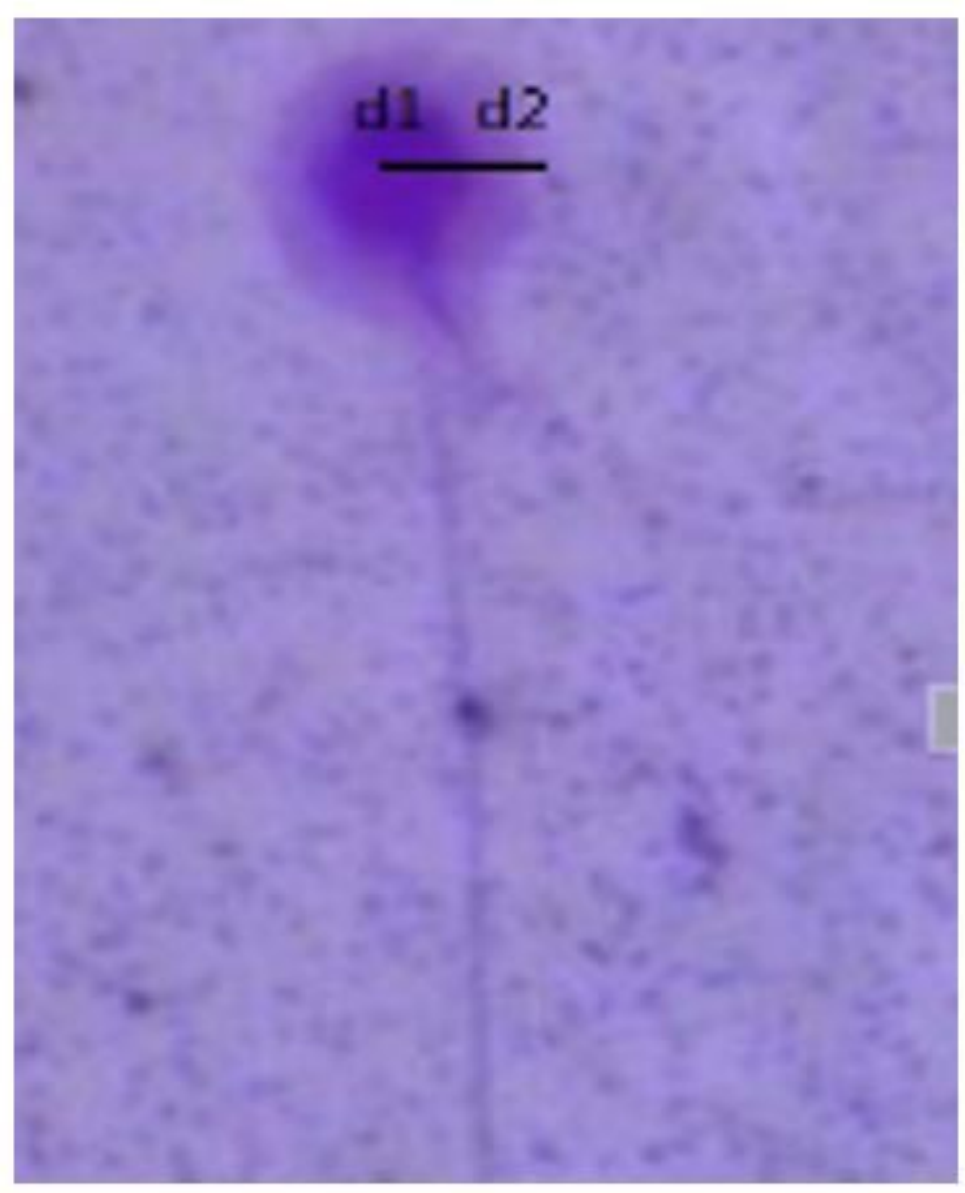

\section{Figure 1}

Schematic diagram of sperm halo 


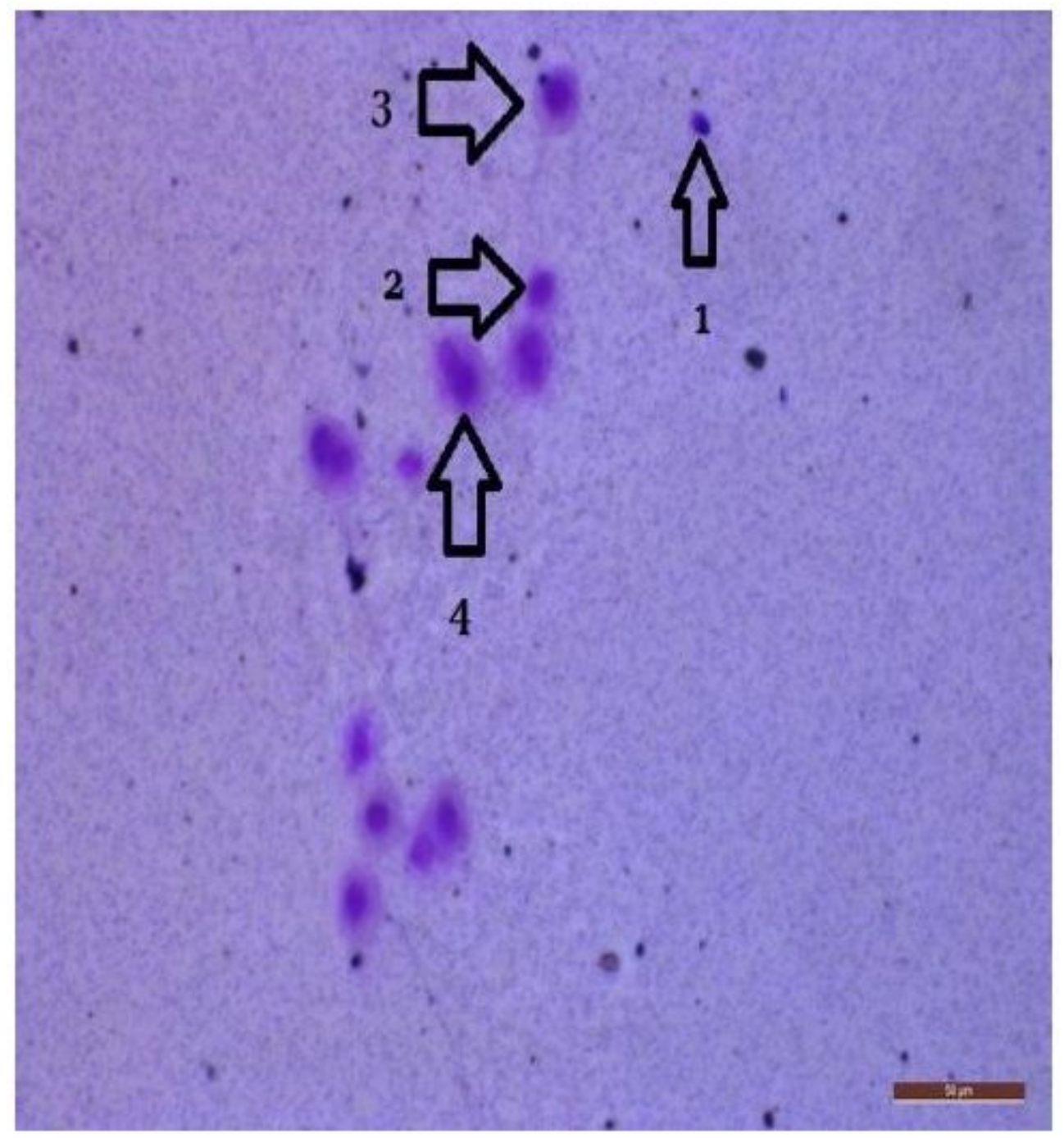

\section{Figure 2}

Results of sperm chromatin dispersion test (40x10) 1: no halo 2: small halo 3: medium halo 4: large halo

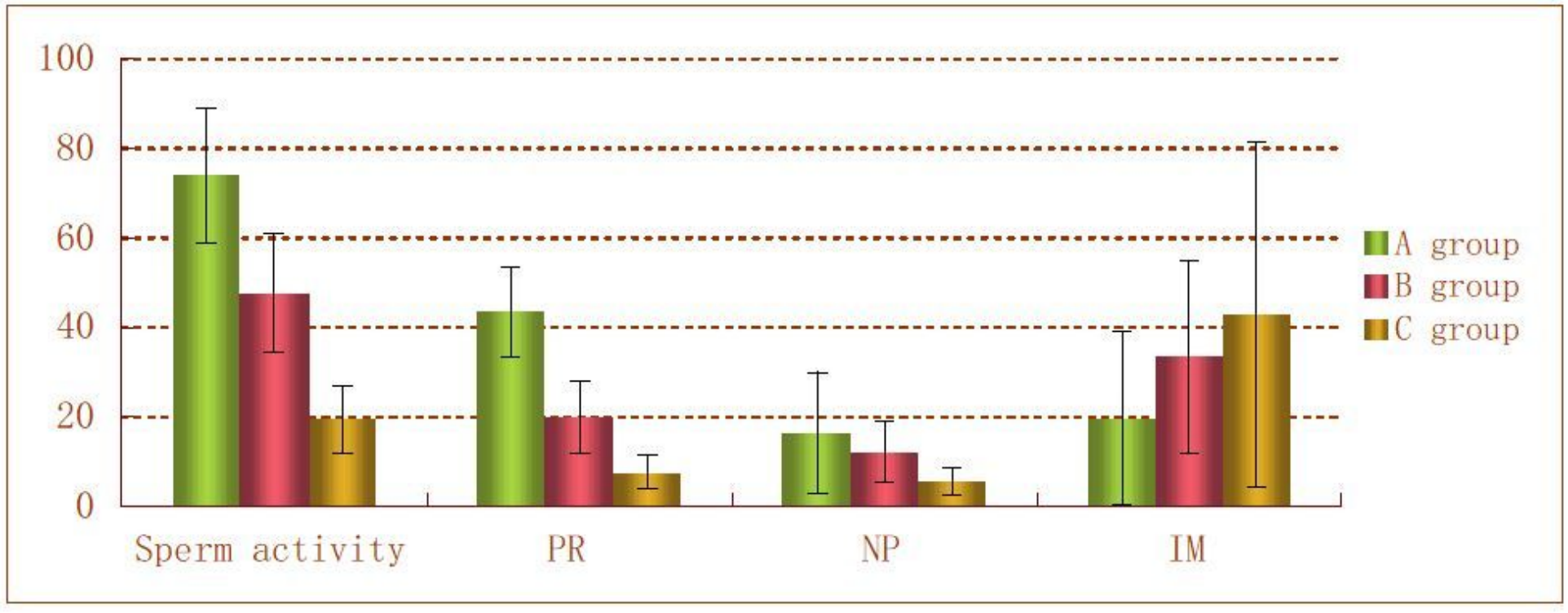

Figure 3 
Semen routine parameters of basic information

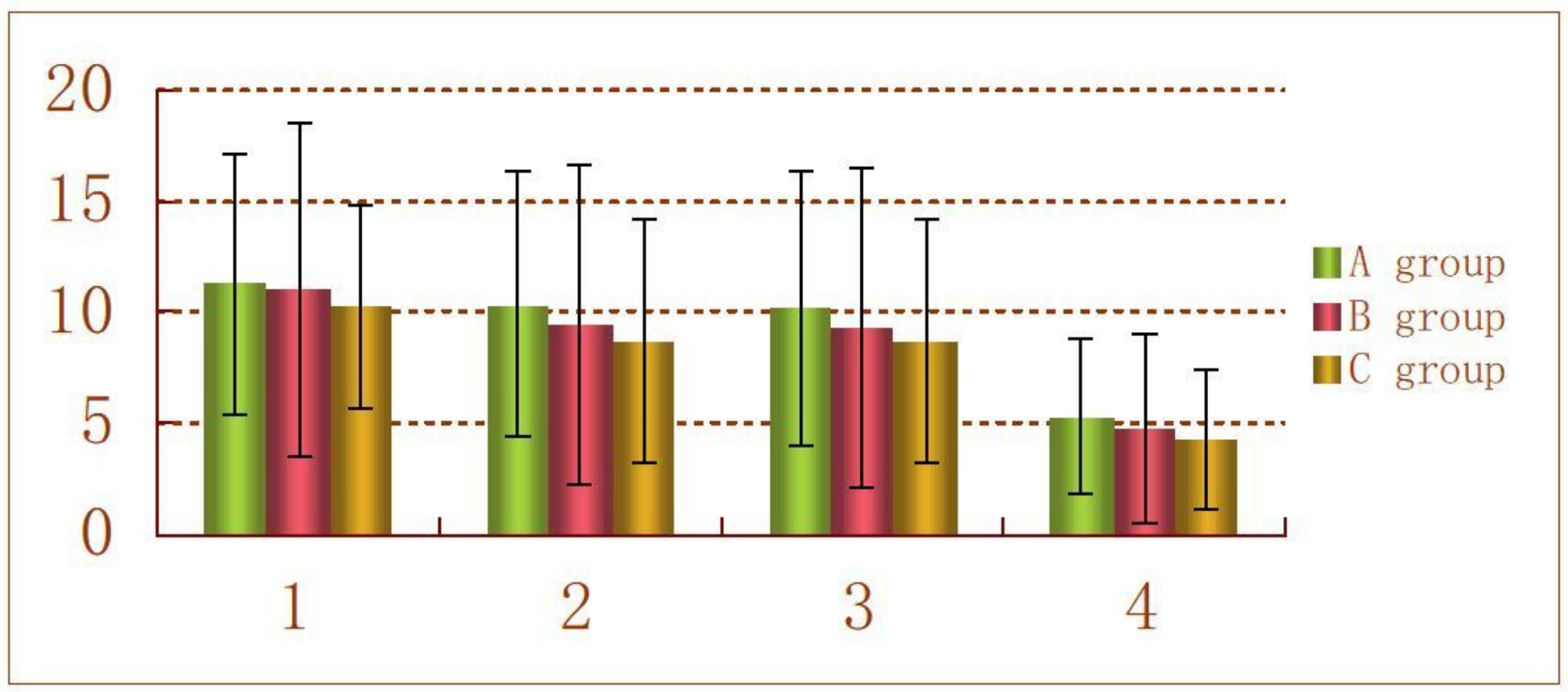

Figure 4

Basic situation each IVF clinical outcome 1. Oocytes number 2. Fertilization number 3. Cleavage number 4. High-quality embryos number 